\title{
Geospatial Analytics for Park \& Protected Land Visitor Reservation Data
}

\author{
Stacy Supak, Gene Brothers, Ladan Ghahramani, and Derek Van Berkel
}

\section{Introduction}

Globally, parks and protected lands (PPL) receive about 8 billion visits annually (Center for Responsible Travel, 2016). The United Nations Environment Programme predicts that ecotourism, nature, heritage, cultural and "soft adventure" tourism will grow rapidly over the next two decades, with global spending on ecotourism expected to increase at a higher rate than the tourism industry as a whole (United Nations Environment Programme, 2011). Therefore, it is increasingly important that PPLs are managed for both the enjoyment of visitors and the protection of natural resources. These sometimes antithetical objectives require managers to strike a balance between visitor impacts and visitor experiences. In this chapter, PPL refers to any natural area that supports recreational tourism on publicly managed land.

Reserving overnight space or access within PPLs helps improve visitor experiences within these natural settings. PPL reservation systems across the globe collect transactional data for reservations at campsites and day-use facilities, as well as permits for the use of trails and backcountry areas. This record of visitation contains information about both destination usage (from the supply side) and visitor behavior (the demand population) (Supak, Brothers, Bohnenstiehl, \& Devine, 2015). Geospatial analysis and geovisualizations produced from these data can be used to promote PPL usage and facilitate sustainable visitor experiences; however, PPL reservation databases are rarely leveraged with these goals in mind (Supak et al., 2015).

At the end of the 1990s, the tourism literature started to acknowledge the benefits of using Geographic Information Systems (GIS) to conduct geospatial analytics and

\footnotetext{
S. Supak $(\bowtie) \cdot$ G. Brothers $\bullet$ L. Ghahramani $\bullet$ D. Van Berkel

North Carolina State University, Raleigh, NC, USA

e-mail:sksupak@ncsu.edu
} 
create geovisualizations for knowledge generation and decision support related to tourism planning and management (Bahaire \& Elliott-White, 1999; McAdam, 1999). At the time, utilization of GIS was rare and awareness of the utility among tourism stakeholders was small. Since then, there has been progress in the use of geospatial analytics to support PPL planning and management, much of which has focused on visitor movement and flow monitoring within PPLs. For example, some studies have utilized GPS visitor tracking to investigate trail impacts (e.g., Beeco, Hallo, English, \& Giumetti, 2013), identify spatial demand and competition for the use of acreage within PPLs (e.g., Beeco, Hallo, \& Brownlee, 2014), issue visitor advisories (e.g., Chhetri, 2015), and make location-specific recommendations to improve visitor activity experiences (e.g., Wolf, Wohlfart, Brown, \& Lasa, 2015). Others have utilized agent-based simulation to better plan for the development of tourist infrastructure such as paths, buildings and viewing platforms (e.g., O'Connor, Zerger, \& Itami, 2005) or to explore the influence of alternative management options on recreationist movement, congestion, and crowding (e.g., Bishop \& Gimblett, 2000).

Tourism and geography researchers have embraced the geospatial nature of PPL visitation. Studies that model determinants of park visits implicate park services, environmental characteristics like topography, climate, proximity to lakes and rivers, and attractive biomes as well as proximity to attractions and amenities (Hanink \& White, 1999; Loomis, 2004; Neuvonen, Pouta, Puustinen, \& Sievanen, 2010; Van Berkel, Munroe, \& Gallemore, 2014). PPL characteristics as well as the quality of those characteristics have been linked to visitation frequency, with parks assessed as "higher-quality" receiving more visitors from a larger geospatial area than parks deemed "poorer-quality" (Hanink \& Stutts, 2002; Hanink \& White, 1999). In addition to considering PPL characteristics, visitors must balance the inherent trade-off between the investment of time, money or effort to achieve travel and the time they can spend at the destination (Mckercher \& Lew, 2003; Neuvonen et al., 2010). Despite the rich amount of geospatial data collected through reservation transactions (e.g., visitor addresses, destination locations) and the clear influence of geospatial factors on visitation, these data remain largely unexplored.

Within the United States and world-wide, government agencies that manage PPLs can benefit from geospatial analytics of reservation data. Data collected from reservation systems are unlike data collected for traditional scientific inquiry, where collection takes place with specific questions in mind (Miller \& Han, 2009). Although these records may not meet the conditions necessary for some types of statistical modeling (e.g., normality or independence), an inductive data mining approach can lead to the discovery of new and unexpected patterns, trends and relationships (Miller \& Han, 2009). Geovisualization of these data sets can be a powerful strategy for initial knowledge discovery because the human brain is extremely effective in recognizing patterns, trends and anomalies. Geovisualizations displaying temporally aggregated and spatially summarized reservation data can be used to support decisions related to management objectives. For example, geovisualizations showing visitor attributes that have been summarized by PPL facility can help managers increase knowledge about the usage of a specific 
facility or group of facilities within a destination region. Examination of the same attributes, summarized and geovisualized by visitor origin can identify which communities are regular users of PPLs, as well as those communities that have historically underutilized recreational opportunities at PPLs. Insight into PPL visitor patterns of demand can help manage, improve and promote visitor experiences for different segments of the population.

Despite the apparent utility of geovisualizing reservation data to address specific PPL challenges, most of these data sets have yet to be leveraged. This is primarily because many organizations find implementing geospatial analytics and geovisualization challenging. The majority of GIS software applications necessary for creating geovisualizations are generic, complicated, and/or expensive; however, a trend is emerging related to the development of innovative and often collaborative, customized web-based mapping applications for creating and sharing geographic information (Haklay, Singleton, \& Parker, 2008). Targeted web-mapping applications reduce the complexity and expense associated with investigating tourism reservation data (S. K. Supak, Devine, Brothers, Rozier Rich, \& Shen, 2014); however, the only demonstration of a web-mapping application targeted for exploring reservation data is limited to geovisualizing the spatial frequency of visitor origins and it does not help users assess the quality of their data, preprocess their data, generate new attributes (e.g., lead-time or distances traveled), or query specific date ranges, destinations or visitor origins. More comprehensive web-mapping applications are necessary to make geospatial investigation of reservation attributes accessible to interested PPL managers, who may have no data management and GIS training. Until these applications are developed, individuals with data management and GIS skills are likely necessary to help PPL managers get the most out of their reservation data.

This chapter describes the value in utilizing spatiotemporal records of PPL visitation from both the facility and visitor origin perspectives. Section 2 of this chapter examines the nature of reservation data, lessons from private sector tourism, as well as the preprocessing, enrichment, and data mining processes. Further, Sect. 2 describes how Federal and local agencies tasked with tourism and resource management can utilize information generated from geographic data mining to geovisualize both PPL demand populations and destination usage. Section 3 introduces an example PPL reservation data set that includes approximately 12.5 million camping, permitting or ticketing reservations made for U.S. Federal PPL facilities during the years 2007-2015, the majority of which are for overnight stays. Section 4 demonstrates specific management decision support knowledge that can be gained through geospatial analytics of U.S. Federal PPL reservation data. Section 5 discusses the future uses of mined and enriched U.S. Federal PPL reservation data. Section 6 presents a brief review of how geospatial analytics of PPL reservation data can be useful in decision support. 


\section{Working with PPL Reservation Data Sets}

Reservation data in their raw form are not entirely informative and often have accuracy issues that need to be resolved before they are useful for spatiotemporal interpretation by managers. PPL reservation data will at a minimum include destination details, visitor details, order dates, start dates, end dates and administrative information (e.g., fees paid). However, depending on setup of the reservation system, different levels of organization and data quality may exist. Destination information could simply be the name of the reserved facility or it could include details about the facility type and location. In terms of visitor details, names, addresses, party sizes, and credit card information is typically collected and stored, but several of these attributes may not be available for analysis due visitor privacy laws. When full visitor origin addresses are unavailable, typically visitor zip codes are still available for analysis.

The level of geospatial precision recorded in a PPL reservation system may vary for both visitor origins and destinations. At the highest level of geospatial precision, a reservation system would record exact geographic coordinates for PPLs and full home addresses for visitors, which can be converted to exact geographic coordinates through a process called geocoding. If a reservation system lacks these highest levels of precision, it still will likely contain origin and/or destination location information in the form of geographic identifiers such as State, Province, Territories, County, or zip code. Any of these geographic identifiers can be used to geospatially locate reservations by visitor or by destination. Lack of a 'geospatial strategy' in record keeping may result in the complete absence of geographic identifiers. If geographic identifiers of visitor origin are not collected within the reservation system, exploration of geospatial demand will not be possible; however, the absence of destination geographic identifiers can be overcome through certain data enrichment processes described later in this chapter.

\subsection{Lessons from Private Sector Tourism}

Traditional application of geospatial analytics for strategic planning in tourism have been limited to corporate efforts (corporate hotel chains, attractions, and resort destinations) and resource supply-chain flows rather than destinations (Chen, 2007). However, increased use of Internet reservation sites has produced large amounts of empirical data that potentially offer rich insight into tourism behavior (Crnojevac, Gugić, \& Karlovčan, 2010). These transactional records are increasingly being used to generate performance metrics. Currently, practitioners in the travel and tourism industry are gaining a better understanding of visitors through the examination of booking rates, stay duration, number in the party and visitor spending. 
While some large tourism companies do their data collection and analytics in-house, other companies, destination marketing organizations and tourism agencies contract with Data-services companies. For example, a state office of tourism can purchase a summary of transactions made within thier state from credit cards with billing addresses outside the state. Using the longitudinal data summaries from the transactions, the tourism managers can identify peak visitation periods, determine origin shares within their destination and relative return on marketing efforts for selected origin markets. These data also can be utilized to identify higherspending visitors and off-season vacancy gaps to fine tune messaging content and timing as well as break into new markets (Lansky, 2016). Knowledge derived from demand population analytics is rapidly replacing the previously relied upon national trend data.

Data-services companies are increasingly providing tourism practitioners with the resources necessary for meaningful spatiotemporal interpretation of visitor data in an effort to create fully data-driven marketing campaigns. These companies are providing destination specific data which include top feeder markets, lengths of stays (duration), booking to arrival time (lead-time), average visitors per booking, search to booking time, visitor spending (from aggregate summaries of credit card transactional information for visitors to their destination) and digital exposure (e.g., media they see before arriving, click through rates of various marketing campaigns) (Lansky, 2016). Many of these companies also break down spending by lifestyle profile. Various data-services companies (e.g., adara.com, airsage.com, buxtonco. com) utilize a mix of Online Travel Agencies, first-party partners, mobile phone data, cookies, and credit card data to deliver their products (Lansky, 2016). As a result, the most comprehensive data-driven support may involve contracting with multiple data service companies.

Methods for gaining knowledge through geospatial analytics and geovisualization of travel reservation data are well established, as the private sector has demonstrated, but these methods are rarely employed for PPL decision support. Without the data that data service companies provide, PPL managers can use in-house collected historical reservation data to identify many of the same destination specific attributes including top feeder markets, lengths of stays (duration), booking to arrival time (lead-time), and average visitors per booking. While corporate decisions aim to increase revenue, these same attributes also can be used to gain knowledge about destination usage as well as geospatial visitor demand, behavior and characteristics. If PPL managers desire this type of knowledge, the raw spatiotemporal reservation data must first be preprocessed, enriched, selected and reduced.

\subsection{Preprocessing and Enriching PPL Reservation Data}

While geospatial attributes available for analysis are determined by the setup of the reservation system, incomplete values within the records due to human error can 
present significant data preparation challenges. Common omissions and domain violations in PPL reservation databases could include visitor zip codes not being entered at all or entered incorrectly (e.g., anything other than a five- or nine-digit number). Non-numeric or incomplete transactional records therefore often need to be preprocessed as the first step toward producing new information. Data preprocessing involves "cleaning" data to eliminate duplicate records and determining strategies for handling missing data fields and domain violations (Miller \& Han, 2009). For example, nine-digits zip codes may need to be reduced to five-digit zip codes. Data preprocessing can be conducted using a scripting language such as python, which is free, but requires coding ability. Alternatively, there are more user-friendly Graphical User Interface options that are open source (e.g., openrefine.org [formerly Google Refine]) or open source with premium support subscriptions (e.g., datacleaner.org). After preprocessing, data enrichment can provide opportunity for richer geospatial analytics. Avenues for data enrichment are detailed in the following three sections.

Generating new meaningful attributes from attributes that exist within the data is a critical step in extracting information and eventually knowledge from reservation data. The following attributes should be created when possible:

Origin-destination location pairs

- The great circle distance traveled between the visitor and the destination

- The least cost road network travel distance for visitor-origin pairs, when the visitor was likely to travel by motor vehicle (e.g., under 300 great circle miles)

- Total number of alternative PPLs that were closer to the visitor's origin than the reserved PPL

- The number of alternative PPLs within a set travel distance (e.g., 50 miles) of the reserved PPL

The date fields:

- The lead-time: number of days between order date and start date

- The duration: end date-start date

- The person-nights: the number of people participating in the reservation multiplied by the duration. This is a measure of the cumulative human occupancy and subsequent cumulative impact to the natural setting

Customer IDs (when available)

- Number of stays in the last year by customer ID

- Total number of nights stayed by customer ID

Destination Clusters (neighborhood groupings of PPL facilities)

- Number of unique facilities in each cluster

- Number of unique agencies in each cluster

- Total number of reservations for each cluster 


\subsubsection{Enrichment from Visitor Origin Geography}

A 10-year study of outdoor recreation visitor behavior in California shows that visitors" "recreation style," defined as gender, age, ethnicity, spoken language, social status, and socioeconomic status influences recreation choice, and attitudes of visitors toward natural resources (Chavez, 2001). Predicting tourist behavior in order to provide better services and protect natural resources through understanding "recreation style" is a fundamental key to successful management actions (Manning, 2014). When visitor origins are available, external secondary data that includes "recreational style" attributes can be joined to each reservation record based on the origin location. The joining of relevant geodemographic data, which estimate the most probable characteristics of people based on the pooled profile of all people living within the area, can be valuable for decision support. When aiming to identify underserved communities, specific attributes such as median age, median home value, median income, and percent of population over 25 years with a bachelor's degree can help explore socioeconomic disparities. Further, these attributes along with observed visitation numbers could be used to model or predict tourist behavior.

"Recreation style" attributes typically are available at some level of geospatial aggregation (e.g., census tract, zip code, county, state, etc.). Secondary geospatial data is increasingly freely available, which can potentially benefit PPL managers that often have budgetary constraints. In the U.S., demographic characteristics describing the residents of a particular area can be obtained for free from the U.S. Census (http://factfinder.census.gov/). Other population characteristics that may help enrich the data such as consumer spending on travel in the U.S. can be found at the Bureau of Labor Statistics (http://www.bls.gov/data/). Proprietary services such as ESRI's business analyst online (http://www.esri.com/software/ businessanalyst/) provide thousands of variables helpful in producing locationdriven market insights that may also help PPL managers better understand their demand populations. If the visitor origin and the order date are both considered, location specific historical data also can be joined to the reservation data set (e.g., gas prices, weather, or terror threat index).

The locational precision of visitor origin in the reservation data set will dictate the resolution of the secondary attribute data that can be joined. For example, if full U.S. addresses for visitors are available, the geocoded points representing visitor origins would need to be spatially joined to the census tracts in which it falls, because that is the smallest discretization of space employed by the U.S. census bureau. If visitor origin data is collected only at the zip code level, it should only be joined to other zip code level data or data that has a coarser spatial resolution such as a county or state. Joining zip code level visitor origins to census track level attributes would not be appropriate. It should be mentioned that associating demographic characteristics to populations within zip codes is not without weakness. See Miller (2008) for a detailed discussion of the benefits and limitations of using zip codes and census block groups for demographic proxies. 


\subsubsection{Enrichment of PPL Destinations Attributes}

External secondary data can also enrich a reservation data set to enhance what is known about each specific PPL facility. This can be accomplished in a few ways. First, demographic data previously utilized in the context of visitor origins or other external data (e.g., historic weather data, remoteness index, etc.) can be joined to specific PPL's based on PPL location, as these attributes might influence reservation choice. Second, attributes describing the amenities or activities offered at a specific PPL facility could be joined to the reservation data, if that data exists electronically in a separate database. Another method for obtaining amenity or activity attributes is through the collection of the content that PPL's advertise on the web. For example, the recreation.gov website is a reservation management system that advertises public-resources for tourism, provides location information for campsites, ticketing and permits, describes the amenities available at these destinations, and provides an overview of destination facilities. This amenity data, when available, can be joined to reservation data either geographically or through common identification codes. Importantly, advertised location data found on web pages can be used to create or quality control PPL facility locations stored in reservation systems or supporting databases.

Automated collection of amenity data is possible using web scraping and web crawling techniques. Web scraping uses an algorithm to access and parse content of a website through an automated process which methodically browses a webpage through the website's url links (Olston \& Najork, 2010; Thelwall \& Stuart, 2006). The consistent structure of website content and organized hierarchies of web pages allows for this systematic information retrieval (Hirschey, 2014). Using such techniques makes it possible to collect large amounts of web content relevant to evaluation of PPL tourism (e.g., amenity details offered by specific campsites).

Implementation of scraping and crawling has become increasingly easy through specialized software. Freely available software (e.g., Kimono, import.io) automatically detect data structure and enable bulk download of website content depending on the web page design. Moreover, scraping software provides a recording interface where the user's actions (e.g., search, page navigation) can be mimicked for complex web searches. While highly user-friendly, such software can be less effective when websites are dynamic (e.g., pop-ups, advertising) or poorly structured. In these cases, coding may be required. High-level programming languages such as Python, Perl and R offer different general and specialized packages that can read and parse text or unicode data for these cases.

\subsection{Data Reduction \& Geographic Data Mining}

Cleaned and enriched reservation attributes are ready to be distilled through data mining practices, so that they may be interpreted within the existing contextual 
understanding (Miller \& Han, 2009). Geographic information is commonly broken into the components of space, time and attribute (Chrisman, 2001). In order to measure one component (time, space, or attribute), one of the other components has to be fixed while the third serves as a control. In classic 2D geovisualizations, time is fixed, space is controlled and attributes are measured. Time is fixed by choosing a specific moment to capture or by aggregating the attribute of interest over some temporal subset of the data. Temporal subsets could be a single day, month, season, or year or the entire time frame over which the data were collected. Selecting temporal periods for aggregation can influence the knowledge discovery process, especially if there are large changes in the attributes over the temporal expanse of a data set. Therefore, management objectives should be considered prior to temporal data subsetting. For example, subsetting the data temporally (e.g., year, season or month) can help identify longitudinal or seasonal changes in visitor behavior. PPL bookings are likely to include temporal trends, such as peaks around holidays, seasonal attractions (e.g., autumn colors of temperate deciduous forests) and 'good' weather that indicate peak tourism demand. Space is then used to control measurement of the attribute for the fixed time frame. PPL reservation data includes spatially explicit origins and destinations, therefore either of these spatial identities could be utilized to control attribute measurement. To measure attributes, an appropriate summarization technique needs to be selected. Standard techniques include reporting for each spatial unit: the count of observations (e.g., total reservation count), the sum of observations (e.g., total number of person-nights) or a descriptive statistics such as median or quantile value (e.g., median distance traveled).

Space, time and attribute decisions for geovisualizations greatly influence what information is communicated. If U.S. zip codes are the most precise geospatial visitor identifier, zip code boundaries that have been drawn to discretize the U.S. will control how the attributes are summarized over chosen time periods. If U.S. counties are the most precise geospatial visitor identifier, county boundaries should be used. If attributes are aggregated or fixed over 1-month time frames, an animation of the individual 1-month geovisualization snapshots could show change over time. Alternatively, including a third dimension in the geovisualization would allow for the temporal aspect of the attributes to be shown; however, depending on the number of records, temporal aggregation may still be necessary, since it is not always possible or useful to render individual tourist activity (Gahegan, 2009). Once space is controlled and time fixed, various attributes can be measured for the entire data set or for a subset of the data. Subsetting the data by some attribute (e.g., visitor origin, PPL facility, PPL facility cluster, lead-time, duration, number in party) can be useful in investigating usage and demand. For example, subsetting the data for a specific PPL facilities or cluster of proximal facilities allows for more precise examination of the geographic dispersion of visitor demand, travel behavior and visitor characteristics for these facilities. Subsetting the data by some property of the PPLs, such as management agency, or specific site type may also provide insight into visitor preference or usage. Regardless of the geovisualization technique employed, distilling data for decision support relies on choosing appropriate 
time frames to aggregate over, spatial units to control the measurements, and summarization of attributes of interest.

\subsection{Utilizing Information Generated from Geographic Data Mining}

Data created during the data reduction and geographic data mining process can be stored in a data warehouse, which in contrast to traditional transactional database design should maximize the efficiency of analytical data processing and data examination to support decision making (Miller \& Han, 2009). Geographic data warehouses are increasingly necessary for the exchange of information through web-mapping services and location-based mobile applications. A good geographic data warehouse should support online analytical processing tools that provided multidimensional summary views of the data. Further, these warehouses can support data cubes, a powerful and commonly applied online analytical processing tool that creates a set of all possible aggregations based on a particular attribute (e.g., person-nights) and some dimension of interest (e.g., PPL destination or month). These aggregations can be over the zero dimension (e.g., total person-nights), one dimension (e.g., total person-nights by PPL destination or total person-nights by month), two dimensions (e.g., total person-nights by PPL destination and month), continuing on the Nth dimension.

If the infrastructure for data warehousing is not available, the tabular outputs of specific data reduction and mining efforts can be loaded directly into a GIS. If the controlling spatial identifier is descriptive (e.g., zip code, county) and does not contain a coordinate set, data containing the same descriptive attributes as geometric objects (e.g., zip code centroid points) are needed for a tabular join. Some geometric object data is freely available from ESRI (ArcGIS Zip Code Layers, 2016). If coordinates can be associated with the reservation data set, most GIS applications can easily allow for the creation of data layers from coordinates. Regardless of the technique utilized to spatially enable the data, the resulting data layers are typically stored as thematic layers where the geospatial location (i.e. zip code centroid point or zip code polygon) is associated with the summarized attribute information (numeric or string type) for use in a GIS (Gomez, Haesevoets, Kuijpers, \& Vaisman, 2009).

Geospatial analysis and geovisualization of PLL reservation data requires strategies for describing visitation trends and tourist behavior that match the needs of administrators. Numerous techniques are available for spatiotemporal data exploration and below we outline several basic, but effective techniques that are viable for PPL management needs. While new sophisticated 3D geovisualization techniques that present the spatial and temporal movements of tourist (Zhao, Forer, Sun, \& Simmons, 2013) have been developed, these type of geovisualizations are most appropriate when tracking a visitors movement within a destination. While 
reservations capture a single tourist's movement from their home to the destination, these paths are usually not complicated, and may not be worth showing.

Basic geovisualizations that presents attribute measurements summarized by PPL facility should display these attributes as points. While basic geovisualizations that presents attribute measurements summarized by visitor origin are most effectively presented as a choropleth map (also called a thematic map) that discretizes the entire area of interest into distinct spatial units (e.g., zip codes). Choropleth maps assign a uniform color or pattern representing a single attribute's value to each spatial unit. The human eye can easily distinguish hot spots and cold spots, so variability in attribute values across geographic regions as well as the level of variability within a region can be easily determined using this technique. When working with U.S. zip codes, a complication arises due to the fact that there are $\sim 40,000$ zip codes and zip code centroids, while there are only $\sim 30,000$ tabulated zip code areas or polygons. This discrepancy results from the fact that $\sim 10,000$ U.S. zip codes have no geospatial footprint because they are post offices or singlesite zip codes (e.g., government buildings, universities). To ensure minimal data omission, reservation attributes should be summarized by visitor origin and first joined to zip code centroid coordinates. Then using a GIS, any zip code centroids that falls within a zip code tabulated area can be spatially joined to that area. Through the spatial join process, attributes can be counted, summed or averaged within the tabulated zip code areas.

\subsection{Geovisualization for Pattern Interpretation of PPL Demand Populations}

In the tourism industry, there are well established benefits associated with geospatial analysis of the relationships of various internal and external data sets for the purposes of better market area understanding and customer profiling (Bell \& Zabriskie, 1978; Elliott-White \& Finn, 1997; Miller, 2008). Since visitation to PPL destinations can be characterized by highly localized utilization, both local and regional utilization and regionally to nationally dispersed utilization with few local residents reserving overnight accommodations (Supak et al., 2015), identifying origin market dispersion is important to characterizing demand populations. Market area definition and subsequent customer profiling of demand populations cannot be accomplished by simply defining distance rings or drive time polygons with respect for each PPL, but rather they should include techniques which account for the geographic dispersion of customers. Examining these dispersions over different fixed time frames allows for investigation of change in demand over time. Alternatively, all records in the enriched data set can be aggregated to identify long-term usage trends for the demand population.

Private sector tourism managers can now pay data services companies to provide historic visitor details including origin markets and other destination specific 
characteristics for characterizing demand populations. The cost of these services could be prohibitive for PPL managers. Fortunately, much of the data assembled by these companies can also be harvested from an enriched PPL data set (e.g., top feeder markets, lengths of stays, booking to arrival time, average visitors per booking). Geovisualizing the top feeder markets is one way to examine the demand population as well as identify underrepresented communities. Identifying underrepresented communities through market definition is desirable for PPL managers who aim to help engage and create our next generation of PPL visitors, supporters and advocates. For example, the Every Kid in a Park initiative in the U.S. provides an opportunity for every 4 th grade student and their family to experience federal public lands in person throughout the 2015-2016 school year. Identifying the underrepresented counties, states and regions through geospatial analytics and geovisualization can help meet the goals of this initiative.

In addition to identifying locations of underserved communities, residents of these locations can be profiled to identify socioeconomic or geographic challenges that might prevent them from accessing public lands for recreational tourism. Tourism marketing has long tracked their customers' characteristics based on their geographic location for the purposes of understanding travel behavior (Miller, 2008; Supak et al., 2014). If visitor demographic and socioeconomic enrichment has occurred, these characteristics can be examined for specific communities as a crude geospatial market profiling technique. There are many examples of more sophisticated market segmentation modeling techniques employed to improve destination marketing such as clustering methods, mixture models, mixture regression models, mixture unfolding models, profiling segments, and dynamic segmentation (Wedel \& Kamakura, 2000); however, simple geovisualizations can accomplish a less formal market segmentation analysis (Supak et al., 2015).

For PPLs within large management systems, reservation data can be leveraged to explore and characterize the demand populations for individual PPL facilities, clusters of proximal PPL facilities or at the whole system level. An effective way to allow managers to geovisualize an individual PPL's demand population would be to create a data warehouse, geospatial data cube and corresponding web-map, so that all reservations for a particular PPL could be queried and demand population characteristics summarized by visitor origin over some spatial unit. Data cubes would need to contain aggregate data across desired dimensions, either produced in advance or on the fly, including standard attribute summaries as well as pointers to geospatial objects (Gray et al., 1997; Miller \& Han, 2009).

\subsection{Geovisualization for Pattern Interpretation of PPL Destinations}

Management strategies for effectively balancing visitor impacts and visitor experiences should include knowledge about the spatiotemporal variations of demand 
population characteristics as well as PPL usage characteristics. When an enriched PPL data set is summarized by PPL facility rather than by visitor origin, geospatial usage patterns of competing use can be explored. Geovisualizations showing aggregated longitudinal destination usage attributes (e.g., person-nights) can provide information to help support management objectives, such as modifying the distribution of visitor impact among facilities within a region. This type of evaluation can be particularly useful when managers want to assess human impact within a region in support of sustainable development and resource allocation decisions. Examining the geospatial relationships of multiple PPL attributes (e.g., total reservation count, lead-time, distances traveled, and duration) for multiple facilities within a specific region can help managers profile the region of facilities as a whole. With this knowledge, availability within the reservation system could be altered, allowing fewer visitors to reserve over-utilized PPLs in favor of neighboring alternative underutilized PPLs. Further, geovisualizations exploring travel distances by PPL can identify PPLs whose demand population is highly localized, both local and regional, or regional to national. If travel distances are small for a specific PPL facility or group of facilities, knowledge of this local utilization can be used for outreach purposes and for improving environmental stewardship.

\section{U.S. Federally Managed PPL Reservation Data Set Example}

To illustrate the benefits that can come for data analytics, and specifically geospatial data analytics, of outdoor recreation reservation data, we examine 12,473,816 reservation records made at 3272 unique U.S. federally managed PPL facilities. The National Recreation Reservation Service (NRRS) maintains a database that collects reservations open-endedly as part of the U.S. Government's Recreation One-Stop Program. Through collaboration with NCSU researchers, the Recreation One-Stop Program management team has made this historic reservation data set publicly accessible (http://ridb.recreation.gov/). Reservations recorded in this database include those for camping, permitting, or ticketing within PPLs that are managed by seven different U.S. federal partner agencies (e.g., National Park Service, Bureau of Land Management, and Forest Service). These reservations encompass both those made ahead of time by prospective visitors and those made by PPL staff at destinations when visitors arrive without reservations (personal communication DeLappe, 2016). Further, not all PPL recreation destinations managed by the federal government are included in the database; some are truly off the grid and have no Internet access. For these PPLs, some allow reservations through recreation.gov, while others do not.

The preprocessing of these data was accomplished with a python script (Python, 2016) that replaced special characters, stripped nine-digit zip codes down to five-digits, added place-holder end dates where none existed, and replaced 
empty field values with NaNs. This data set includes records for order dates that range from January 1, 2007 to December 30, 2015, with start dates that range from January 15, 2007 to December 29, 2016. While no records were found to be missing order date or start date values, end date values were missing from 300,804 records. Further, 282,857 records have an order date after the start date. These two values may be related, as many PPLs allow for payment after hours through a drop box and these reservations are then entered into the system after the start date (personal communication DeLappe, 2016). Another 2,563,418 reservation had the same order and start date, many of which were likely booked by PPL employees when visitors arrived at the destination without a reservation.

Figure 1 shows the percent of total reservation records (January 1, 2008December 31,2015$)$ that were ordered or started on each day of the year. Data from 2007 was not included in this figure, as many reservations starting in 2007 were ordered in 2006. Order date spikes on the 15th of the month from December to April correspond to Yosemite National Park releasing camping blocks on those days (personal communication DeLappe, 2016). The general trend of the graph shows increasing utilization from the beginning of March to mid July, with decreasing utilization from mid July to the end of October. The U.S. Federal holidays of Memorial Day, Independence Day and Labor Day (the last Monday in May, July 4th, and the first Monday in September, respectively) each capture almost $1 \%$ of the total reservation start dates. The order date dip around the 4th of July Holiday suggests recreation is occurring, keeping prospective visitors away from the reservation system.

Of the 3272 unique facilities, only 31 offer ticketing (e.g., White House Easter Egg Roll), which is likely not for overnight activities. Both overnight and day-use permits are offered at 51 facilities. While ticketing and permitting reservations may not necessarily include overnight stays, these reservations have not been removed from the data set used for this analysis. Given that there are 3190 facilities $(97.5 \%)$

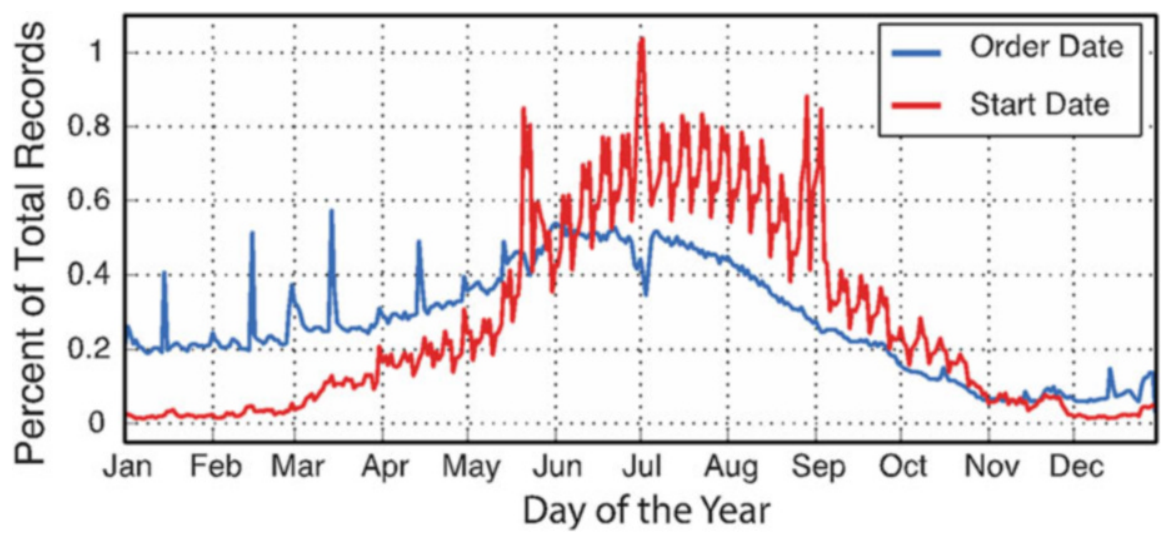

Fig. 1 Percent of total reservations for U.S. Federal PPLs whose order or start date was between January 1, 2008 and December 31, $2015(\mathrm{n}=12,072,092)$ binned by day of the year 
that provide overnight camping accommodations, it is fair to assume that most reservations represent overnight stays. Of the seven agencies that provide these services, five agencies offer more than two camping destinations and they include the US Forest Service (2233 unique facility names), the US Army Corps of Engineers (697 unique facility names), National Park Service (217 unique facility names), Bureau of Land Management (28 unique facility names), and the Bureau of Reclamation (11 unique facility names).

Fields provided in these historical data that pertain specifically to the destination include facility name, facility, agency, site type, facility zip code and facility coordinates. Of the unique facilities in the data set, many were missing zip code values, but only five were missing coordinates or had discord in coordinate values across the records. These facilities were manually searched and coordinates replaced for 7403 records associated with these five facilities. This led to $100 \%$ geolocation of the facilities; however, inaccurate coordinates may exist within the PPL database. It should also be noted that these facility geolocations are far more precise than previously published facility locations from the same reservation system (Supak et al., 2015), as these geolocations were previously based on facility zip code centroids and not facility coordinates. This increased precision in PPL location allows for examination and comparison of specific facility attributes (e.g., total reservation count, median lead-time, median visitor travel distance) within a geospatial neighborhood, which was previously not possible.

For the demand population, approximately $98 \%$ of reservations included a fivedigit zip code of visitor origin. Visitor zip codes were geolocated by joining each record's visitor zip code to the known latitude and longitudes of the zip code centroid. To ensure the highest percentage of geolocation of visitors, a commercial zip code database was used (http://www.zip-codes.com/zip-code-database.asp), which contained 40,145 zip code coordinates. A tubular join of these data led to $96 \%$ of the reservations being successfully geolocated for visitor origin. With both destinations and visitor origins geolocated, enrichment from attributes within the data set commenced. Using the origin/destination location pairs the great circle travel distance between the visitor and the destination were calculated.

In order to better understand the spatio-temporal relationship between distances traveled and seasonality, Figure 2 presents a graph of the distances visitors are willing to travel based on the start date of the reservation. Quantiles show the distribution of travel distances for all records on a particular day. The overall trend of the graph shows that for all start dates, $50 \%$ of the reservations are made for parties traveling less than $200 \mathrm{~km}$ for the experience, with virtually no weekend, seasonal or holiday effects. Examining the $75 \%$ quantile shows start dates between April 1 and November 15 have little variability in the distances traveled (between $200 \mathrm{~km}$ and $400 \mathrm{~km}$ ), with a small weekend effects becoming visible. For start dates between mid November and the end of March, $25 \%$ of the reservations (still the $75 \%$ quantile) were for parties that traveled more than $1000 \mathrm{~km}$, with a less regular weekend effect than the rest of the year. This indicates parties are more willing to travel farther distances for recreational tourism in the winter than in other seasons, perhaps for winter specific activities such as back county skiing. When examining the $90 \%$ quantile, the weekend travel distance signal and winter seasonal effect 


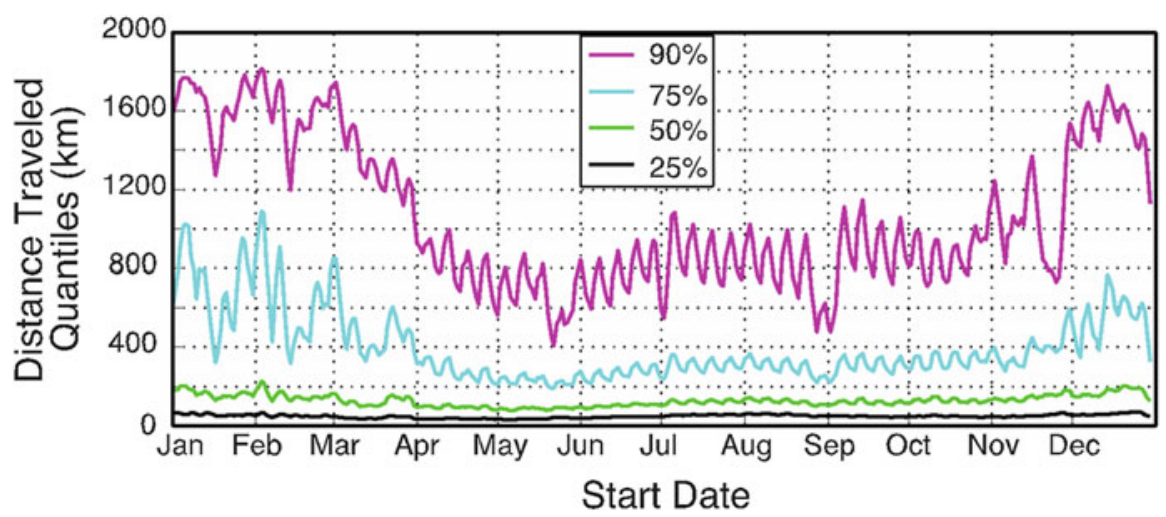

Fig. 2 Great circle distance traveled between visitors and U.S. Federally managed PPL's with start dates between January 1, 2008 and December 31, $2015(\mathrm{n}=12,072,092)$ binned by day of the year

become more pronounced. Further at the $90 \%$ quantile, distances traveled drop for Memorial Day, Independence Day, Labor Day and Thanksgiving holidays start dates.

Further enrichment from attributes within the data set produced the attributes of lead-time, duration of stay, and person-nights (a measure of cumulative nights of human occupancy). Enrichment from visitor origin geography and PPL name are not presented in this chapter, but will be considered in future analysis. The leadtime attribute is explored to uncover national trends in temporal reservation making. Figure 3 shows lead-time quantiles for the reservations based on start date. The overall trend of the graph shows that lead-time generally increases in the summer months and for most start dates, $50 \%$ of the reservations are made less than a week prior to the start date. The spikes in the lead-times across all quantiles for the U.S. Federal holidays of Memorial Day, Independence Day, Labor Day and Thanksgiving (fourth Thursday of November) indicate visitors make travel plans for these holidays far more in advance than for other times of the year. These U.S. federal holidays, in addition to Christmas and New Years also are the only start dates for which the $25 \%$ quantile of lead-time is greater than zero. The spacing between the quantile curves within the figure can be used to gauge temporal changes in reservation booking rates. Uniform spacing indicates temporally steady booking rates, while gaps or closures signify non-uniform booking rates. The tighter the curves, the less lead-time variability observed. Notice the tightening of the curve between the $90 \%$ and $75 \%$ quantiles in March. Of reservations made for March start dates, $90 \%$ are booked within 3 months of the start date. This indicates that March visitations are planed less in advance than other months. 


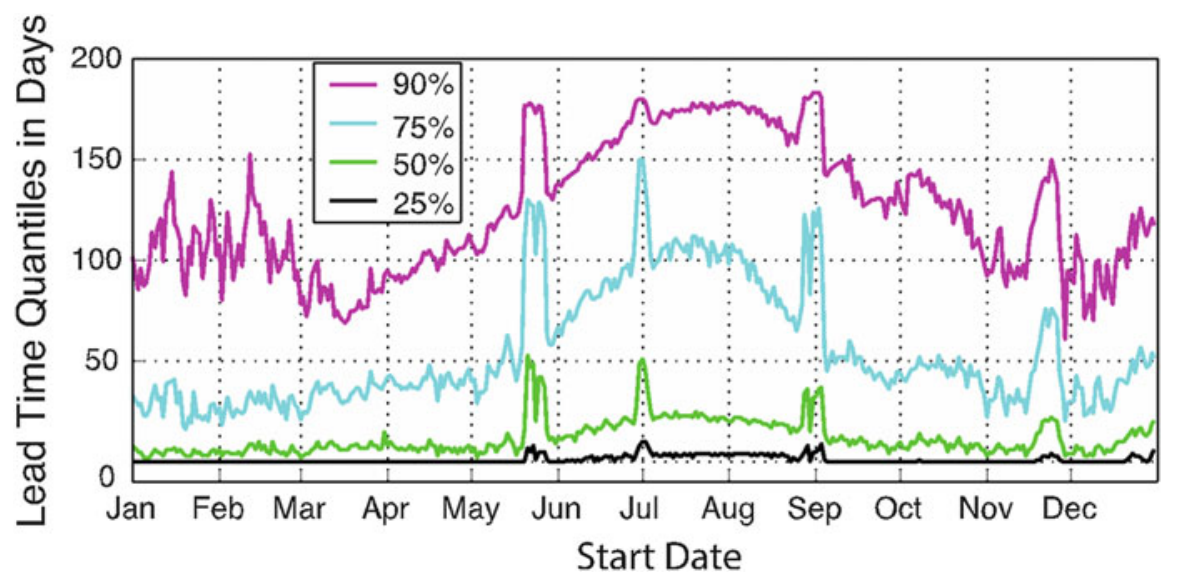

Fig. 3 Lead-time between order date and start date for U.S. Federal PPL reservations with a start dates between January 1, 2008 and December 31, $2015(\mathrm{n}=12,072,092)$ binned by day of the year

\section{Geovisualizations for U.S. Federally Managed PPLs}

The geovisualizations presented in this section examine national trends in the 9-year U.S. Federal PPL reservation data set, which include all records whose origin-destination pairs could be geocoded $(n=12,003,292)$. While these data could be subset for a particular PPL or group of PPLs, season, or visitor origin region, as described previously in this chapter, the complete data set is considered for this demonstration of the value of geovisualization in PPL decisions support. Figures 4, 5, 6, and 7 present enriched reservation attributes that have been summarized (controlled) by visitor origin zip code. These geovisualizations explore the demand population including an index of national utilization, the median travel lead-time in days, the median travel distance, and the distance difference between the 25th and 75th distance quantiles, which helps describe the distance distribution curve for each origin zip code. Figures 8 and 9 present enriched reservation attributes that have been summarized (controlled) by PPL name. These geovisualizations explore the distribution of total reservation count across the U.S. and a comparison of utilization attributes for distinct facilities located within the Ashley National Forest.

All geovisualizations presented in this section were generated using ESRI's ArcGIS for Desktop10.3.1 (ArcGIS for Desktop, 2016); however, similar figures could be produced with open source GIS applications (e.g., QGIS). The quantile style classification methodology employed (Figs. 4, 5, 6, and 7, 9) aims to put an equal number of unique ZIP codes into each of five categories based on the summarized attribute value. Figure 8 employs a natural breaks classification method because the quantile method was not appropriate due to three reservation count outliers identified in a univariate graph for total reservation count. 
In order to better understand national level utilization of federal recreational opportunities, a national index of federal land utilization was generated using the total sum of all person-nights for the 9-year data set $(151,655,333)$. The index simply normalizes the total sum of all reservations person-nights per zip code by ESRI's 2014 total zip code population estimate (Fig. 4). Another way to think about this index is that it captures the cumulative nights of human occupancy and subsequent cumulative impact to the natural setting. A zip code with a Federal Land Utilization index of one would result if each person residing within the zip code made exactly one reservation for one person for one night during the 9-year period. However, it is likely that repeat visitations account for many of the reservations. Without access to customer identification data, the percentage of repeat visitation cannot be determined.

Figure 4 reveals that the south-central Midwest, much of the West coast and pockets along the Appalachian Trail exhibit the highest utilization indices within the contiguous U.S. This means that per capita, residents from these regions make reservations on federal lands more often (red in Fig. 4) than those within other regions. In general, Indiana, Ohio, Eastern New York, costal Carolinas, Louisiana \& Western Mississippi underutilize federal lands relative to the national population. This decreased demand may be a function of the lack (or limited availability) of federal PPLs in this region (Figs. 6 and 8); however, alternative reasons such as lack of interest in visiting federal lands, propensity to stay on private or state land for overnight stays, or general disinterest in outdoor recreation require further examination.

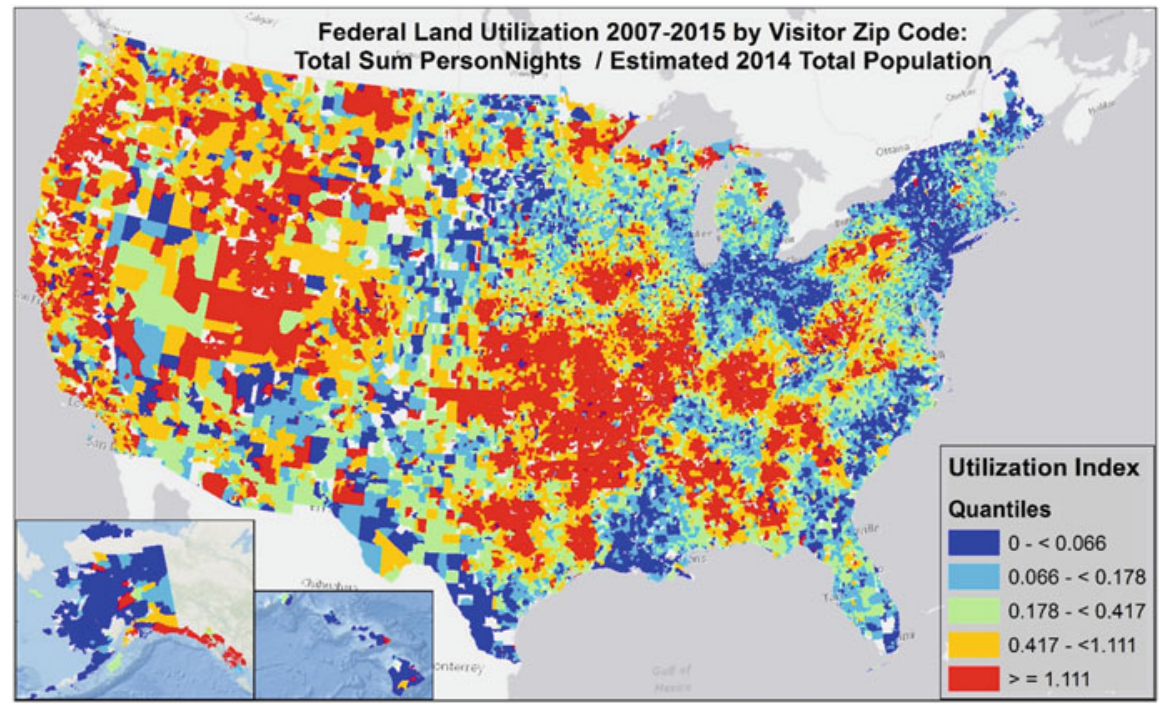

Fig. 4 Federal land utilization index by visitor zip code derived from the total sum of personnights at U.S. federally managed destinations over 9-years (2007-2015) normalized by the estimated 2014 total zip code populations 


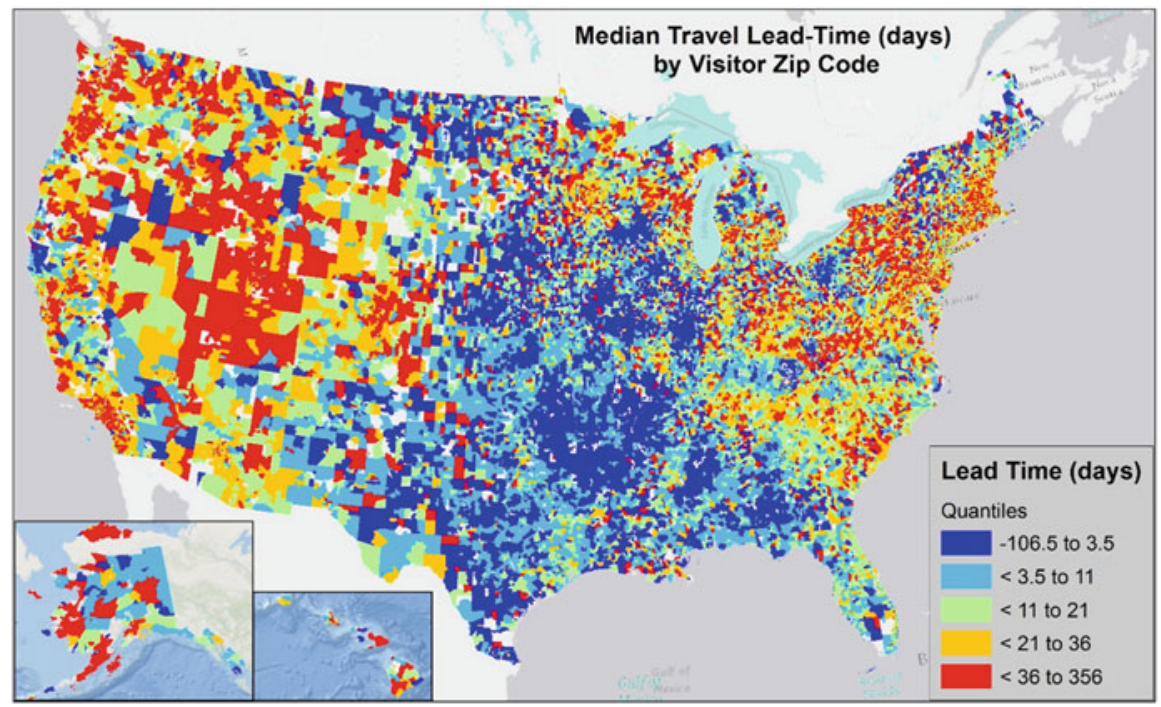

Fig. 5 Median travel lead-time in days when booking U.S. federally managed destinations by visitor zip code, 2007-2015

Next, the median lead-time is investigated (Fig. 5) as the summary statistic rather than the average lead-time because the lead-time distribution for the NRRS data set was not normally distributed. Locations where higher utilization is observed in Fig. 4, largely correspond to locations with short median lead-times. In particular, Arkansas and portions of the states immediately surrounding Arkansas have short median lead-times. Parts of New England and out West, specifically Utah have longer median lead-times. Geovisualization of the 25th and 75th quantiles for travel lead-time (not shown here) also display similar patterns.

Figure 6 presents the median great circle travel distance between visitors and destinations by visitor origin. The median travel distance was selected as the summary statistic rather than the average travel distance because the distance distribution for the NRRS data set was not normally distributed. Visitor travel for recreational tourism on federal PPLs east of the front range of the Rocky Mountains is typified by low median travel distances for zip codes (medium to light blue in Fig. 6) immediately surrounding the facilities. In many cases, a facility or group of facilities become the bull's-eye or locus at the center of concentric circles whose median travel distance increases with increased distance from the facility (dark blue to medium blue to green). This suggests that residents proximal to facilities are in fact reserving local facilities for recreational tourism. Moreover, this pattern of localized utilization is very similar to the utilization pattern shown in Fig. 4. Visitor travel for recreational tourism on federal PPLs west of the front range of the Rocky Mountains is typified by a less concentric bull's-eye pattern, but there is still some local utilization. Regions with few or no federally managed PPLs (e.g., Indiana), not surprisingly have higher median travel distances. However, many PPLs are surrounded by zip codes whose residents travel the farthest, such as in New England, Florida, Southern New Mexico/West Texas, Michigan and Ohio. 


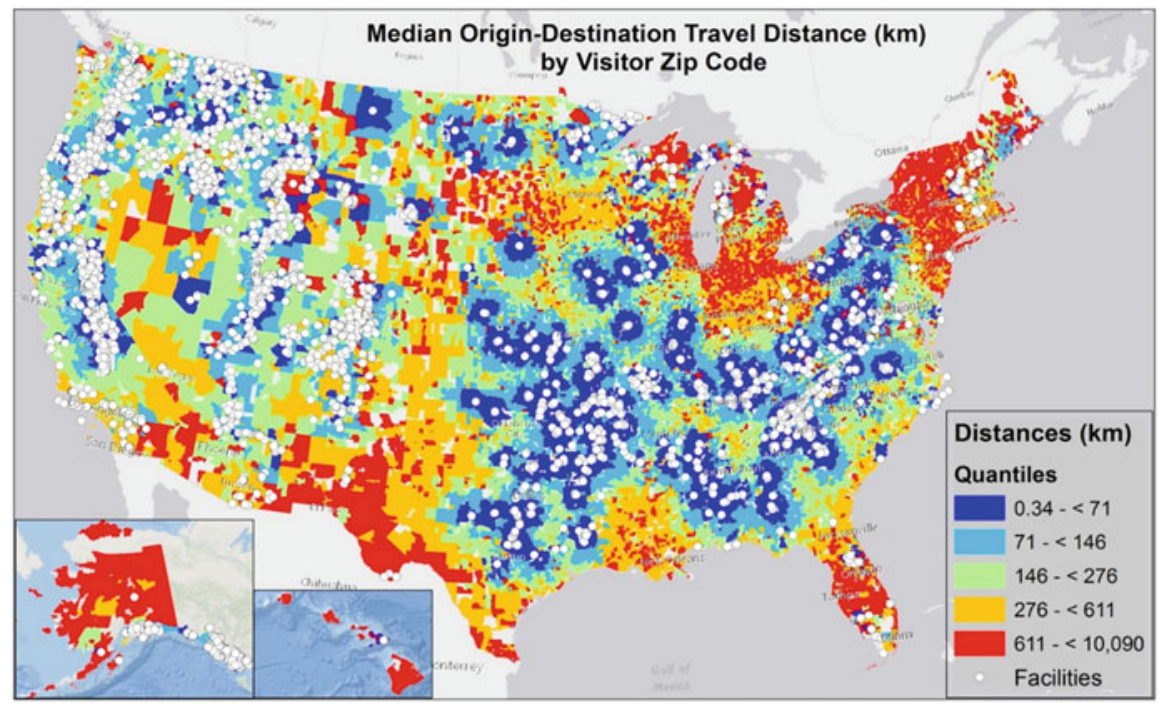

Fig. 6 Median origin-destination travel distance to U.S. federally managed destinations by visitor zip code, $2007-2015$

In order to further examine the origin-destination travel distance distribution curve for each origin zip code, for which the median values were presented in Fig. 6, the difference between the 25th travel distance quantile and the 75 th travel distance quantile is geovisualized in Fig. 7. If the difference is small for a specific zip code, the origin-destination travel distance distribution curve will be a very tight spike, indicating many visitors (the middle $50 \%$ of visitors) travel relatively similar distances from their origin to their reserved destination. If the difference is large, the origin-destination travel distance distribution curve will be broad and flat, indicating larger variability in the distance visitors are willing to travel to their reserved destinations. The distance differences values measure the range over which visitors from the same origin zip code are wiling to travel, which indicates similarity or dissimilarity in destination preference. For example, if a region is dark blue in both Figs. 6 and 7, then residents of that region make reservations that are highly localized with little variability in the distance visitors are willing to travel to their reserved destination. If a region is light blue in Fig. 6 and red in Fig. 7 (e.g., parts of New England), visitors are utilizing local and regional PPLs, but there is large variability in the distance visitor are willing to travel to their reserved destination and larger distances traveled in general.

Geospatial demand can also be examined from the supply side by looking at enriched reservation attributes summarized by U.S. PPL destination. Figure 8 explores the distribution of total reservation count at federally managed PPL destinations across the U.S. In general, west of the front range of the Rocky Mountains is typified by an abundance of PPLs, many of which have relatively low total reservation counts. Relatively higher total reservation counts can be found 


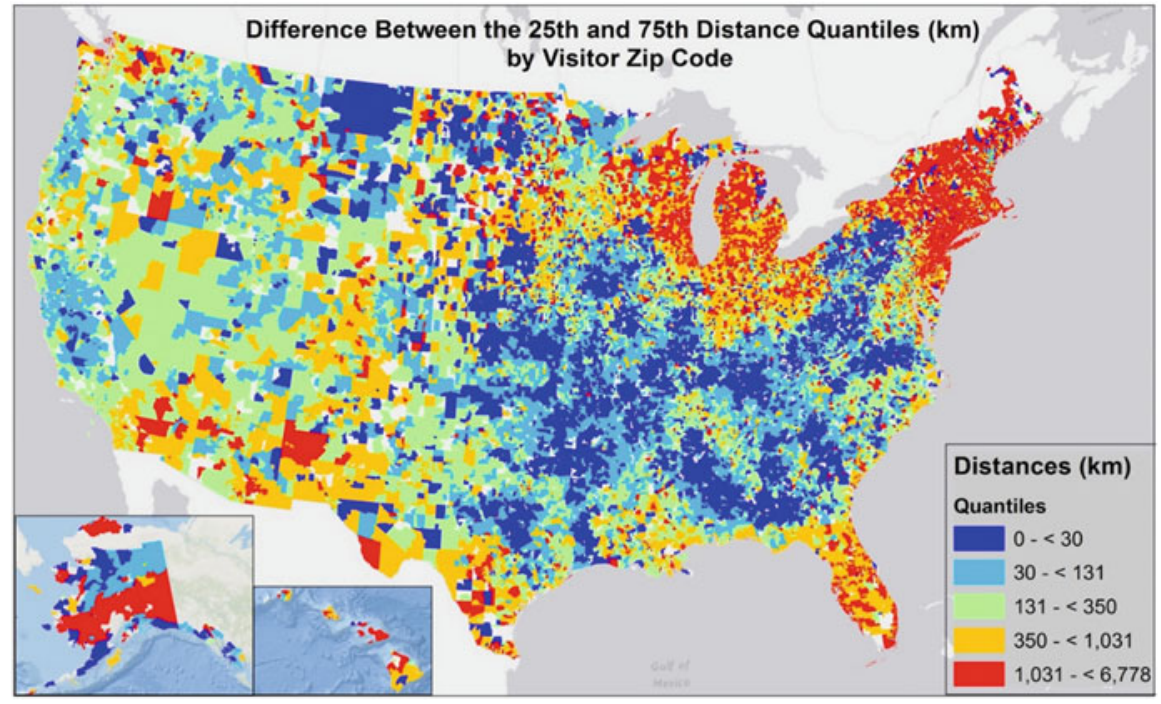

Fig. 7 Difference between the 25th and 75th travel distance quantiles for U.S. federally managed destinations by visitor zip code, 2007-2015

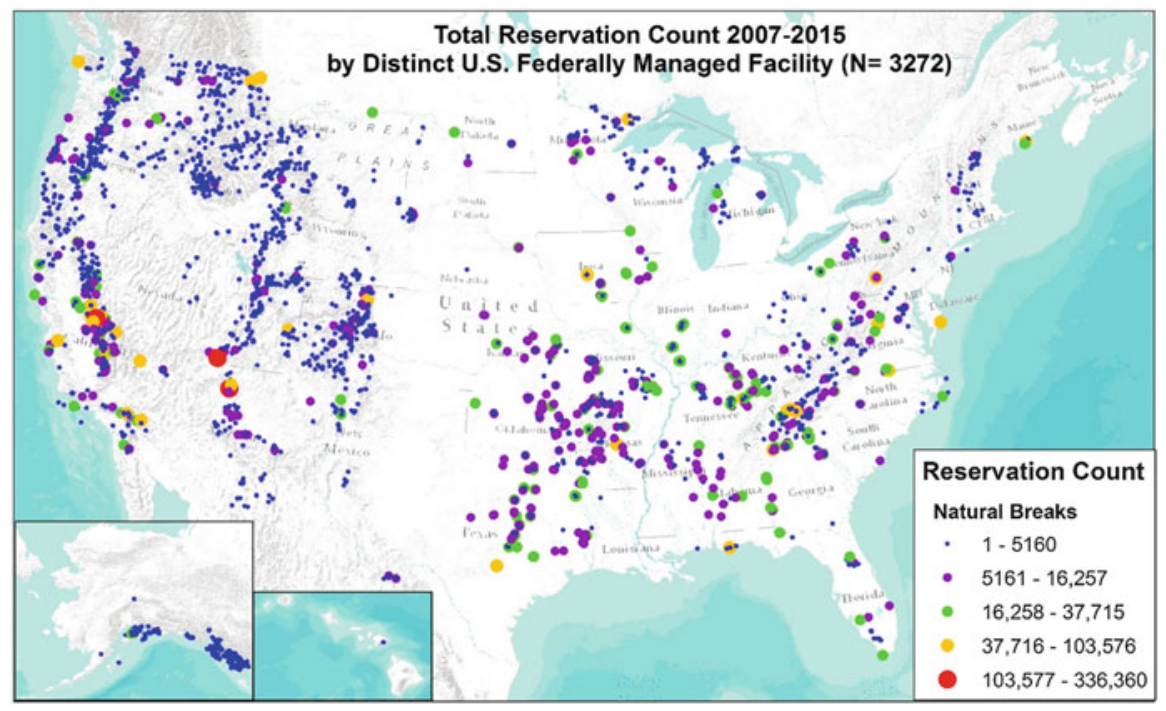

Fig. 8 Total reservation counts for U.S. federally managed facilities, 2007-2015

for PPLs in the eastern U.S., particularly for those PPLs that were at the center of the distance traveled bulls-eyes (Fig. 6) Three unique facilities stand out as having the greatest number of reservations: Mather Campground, Grand Canyon National 
Park, 336,360 reservations; Upper Pines, Yosemite National Park, 201,174 reservations; and Watchman Campground, Zion National Park Service, 182,201 reservations.

While the previous national maps provide an opportunity to explore regional distribution patterns, PPL managers also can learn about the utilization of specific facilities within the context of their neighborhood cluster. Figure 9 provides a comparison of demand for all facilities clustered within the Ashley National Forest region near the Green River in northeastern Utah. This figure provides a comparison of the median distance traveled, median lead-time of the reservation, person-nights, and total reservation count for 16 distinct facilities within the reservation system. Median travel distances are quite similar across the 16 facilities and range from $191.8 \mathrm{~km}$ to $225.7 \mathrm{~km}$. While one facility does make it into the top national median travel distance quantile, all other facilities in the area fall into the second highest quantile. This likely means that visitors to this destination region are drawn from the same origin markets. The median lead-time for reservations is much more diverse than the travel distance among these facilities, with a high of 360 days lead-time and a low of 19 days. This diversity indicates the varied demand for these facilities (i.e. high demand with high lead-time), which could reflect either or both the attractiveness of the facilities or restricted opportunities (limited availability of reservations).

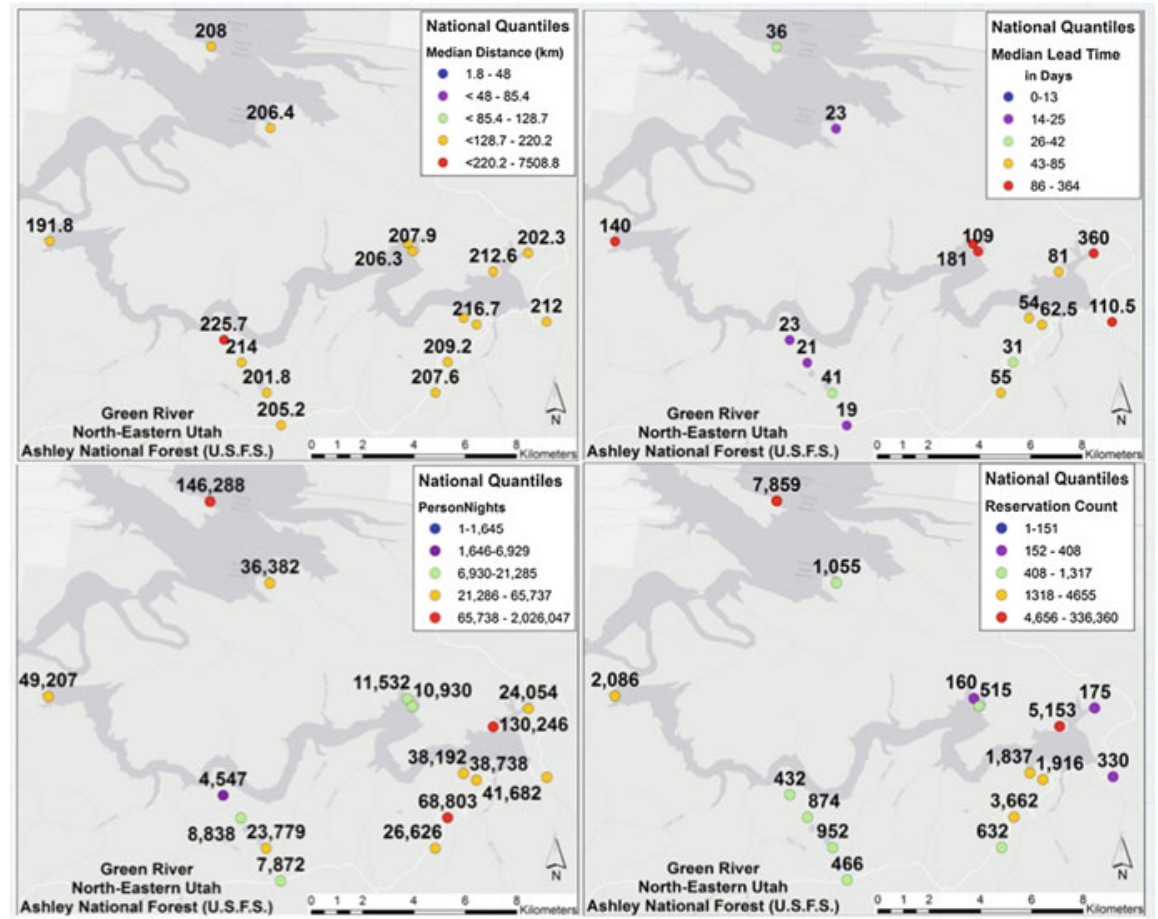

Fig. 9 Reservation attributes for 16 facilities within the Ashley National Forest including distance traveled, lead-time, person-nights, and total reservation count, 2007-2015 
Comparing person-nights, a measure of cumulative human occupancy and impact, among the facilities can inform managers about relative demand within the region or for specific facilities. Person-nights for these facilities range from a low of 4547 to a high of 146,288 . The facility with the lowest value may have the lowest demand or the site itself may require limited use. This facility had the highest median travel distance for all facilities in the cluster; so, visitors are willing to travel a longer distance for that facility. This observation along with the low leadtime for this specific facility may be related to settings within the reservation system. Additional information about the capacity of this facility and its reservability are necessary to better interpret this geovisualization. Finally, the total reservation count for the region and individual facilities within the region can also inform managers of total visitation demand. The range of the total reservation count for these facilities is from 160 to 7859 .

Examining indicators of demand from the PPL perspective (e.g., Fig. 9) can help support management objectives such as increasing knowledge about managed PPLs and supporting sustainable development. Within the Ashley National Forest, the metrics presented indicate that individual facilities have different use load/capacity, as four of the five facilities with highest reservation lead-times (most popular) have the lowest number of reservations (limited or restricted number of opportunities), which creates a high demand. This distribution of demand among facilities could also provide managers knowledge with which they can inform potential visitors of alternative options available or change availability in the reservation system to more equitably distribute use (spatial and temporal) among the facilities within the region.

\section{The Past, Present \& Future of U.S. Federally Managed PPL Reservation Data}

In 1998 the Recreation Information Database (RIDB) was created to provide a web-based resources for citizens interested in accessing supply-side data including recreation area and facility data (Recreation Information Database-RIDB, 2016). In 2006, the newly created Recreation One Stop program integrated RIDB data into an inter-agency recreation reservation website (www.recreation.gov) in an effort to provide visitors a more comprehensive resource. At that time, the RIDB data was still available to third parties, but it lacked site-specific details that were included in the website Recreation.gov. In 2014, the RIDB implemented an XML feed from Recreation.gov to pull in all site-level data as a way to provide a more complete supply-side recreation data set.

With passage of the Digital Accountability and Transparency Act of 2014 (DATA Act), there are now legal requirements to provide open data and many federal agencies are working to overcome the challenges associated with sharing data (Panel Discussion on Changing the Culture for Open Data, 2015). In 2015, 
RIDB developed and deployed an application programming interface (API) to provide data in additional formats (e.g., JSON \& CSV) as well as ways to filter or subset recreation supply side data by specific state, activity or organization (Recreation Information Database-RIDB, 2016). On February 10th, 2016 at the requests of NCSU researchers, access to historical reservation data was added to RIDB website as downloadable annual CSV files (2007-2015). Recreation One Stop program staff aim to have the historical data available through the API shortly (personal communication DeLappe, 2016). Providing access to this historical data in open, machine-readable formats, in compliance with President Obama's executive order, is a great example of how government programs can overcome the legal, technological, and cultural barriers that sometimes limit adoption of open data standards.

The geospatial analytics and geovisualization techniques presented in this chapter are one approach for transforming flat CSV files containing historical reservation data into information that supports management decisions. A more effective way to achieve this goal and to promote the use of this open data may be to create a direct link between the reservation system and a historical reservation data warehouse, so that as reservations occur they could automatically be preprocessed, cleaned, and enriched. Geovisualization then can be accomplished through the development of web-based mapping application that utilize simple and customizable APIs, such as Google Maps ${ }^{\mathrm{TM}}$ API. One large benefit of utilizing APIs and supporting data infrastructure (e.g., data warehouses and data cubes) for web-mapping application development is that users are provided with accurate real-time data, from which they can extract timey actionable intelligence. These web-maps could be designed and developed for specific management objectives or for perspective-visitor travel planning decision support.

From the management perspective, the U.S. Department of Agriculture's Animal, Plant, Health Inspection Service managers have begun using reservation data to geovisualize the origins of visitors who camp at specific facilities, so that they can track infected firewood dispersion (personal communication DeLappe, 2016). This is just one example of how the geospatial component of reservation data can be used to help managers provide better overall experiences to visitors. Further, historical reservation data can provide decision support knowledge relevant to complex PPL ecosystems that need to be carefully managed with regard to decisions about invasive species, climate change, and development around and within these sites as well as other management issues. For these reasons, agencies tasked with controlling invasive species or the spread of plant and tree diseases, would greatly benefit from the real-time information offered by web-maps.

While this chapter has focused primarily on geospatially examining historical recreational tourism reservation data for improved decision support by managers, it also should be mentioned that allowing the public easy access to historical usage geovisualizations could help support perspective-visitors as they plan travel. In an effort to bring our nation's PPLs closer to the people, to which they collectively belong, the Recreation One Stop program held a developers summit on April 11-12, 2015. The invitation outlined the benefits of bringing "wild areas" within 
closer reach of the people including improved public health and stress reduction resulting from exercise and time spend outdoors, increased awareness of man's impact on nature and general ecological principles, and increased connections between more citizens and our country's diverse history (myAmerica Developer Summit, 2015).

Among the summit sessions, several could have utilized the geospatial aspects of the historical reservation data. For example, one session described using Census Data to discover communities of interest related to the Every Kid in a Park. These discovered communities could be refined further by eliminating communities that are well represented in the historical reservation record. Another session yielded a prototype that allowed prospective visitors to access spatiotemporal historical reservation data for the Grand Canyon to assist in trip planning, and specifically for identifying the most historically crowded times. This prototype could be extended to allow potential visitors to compare PPLs within desired destination regions or over different date ranges. Prospective visitors could then consider visitation rates as they decide when and where to visit, which could ultimately lead to improved visitor experiences and hopefully a new generation of PPL enthusiasts.

\section{Conclusions}

Data analytics is booming across domains, industries and data platforms. In the travel and tourism domain, analysis is largely focused on measuring and capturing the tourism experience. To this end, the geospatial component of visitor experience is captured with increasing detail about demand populations (e.g., geo-tagged tweets, Facebook posts, etc.). Increased use of Internet reservation sites has produced large amounts of geospatial data that potentially hold a wealth of information about historic usage, which can be used to the benefit of tourism destinations. The private sector is using these transactional records to generate performance metrics, many of which are highly geospatial such as origin shares within a destination, relative return on marketing effort or geodemographic customer profiling. PPLs can also gain a better understanding of visitors through geospatial examination of metrics such as booking rates, stay durations, lead-times and the number in the party. This information is useful for PPL managers who are tasked with balancing the needs as well as the impact of visitors.

Knowledge discovery from geographic data that is collected without end, such as from a PPL reservation system, is nontrivial and requires distinctive consideration and techniques (Miller \& Han, 2009). The inductive approach of mining enriched PPL reservation data sets described in this chapter can serve as a guide for creating historical usage knowledge. To maximize the inductive data mining approach and increase the opportunity of finding relationships, PPL reservation data sets should be enriched from attributes within the data set as well as from relevant external data. Geovisualizations of these mined and enriched data can be 
an effective means of communication and can deliver insights that might otherwise be lost, as they can present new and unexpected patterns, trends and relationships. These insights can lead to improved management decision support.

Globally, any agency tasked with tourism and resource management at any level of government (i.e. Federal, State, Province, Prefecture, County, etc.) can use this approach to examine patterns and trends within the empirical evidence created though reservation booking. Researchers supporting effective recreational tourism management also can use this approach to modify or formulate new hypotheses, which can in turn help modify existing theoretical frameworks related to tourism and recreation. Specifically, knowledge gained from PPL reservation data can help generate new theories related to distance decay, origin markets, or set choice. These theoretical constructs support more robust data analytics practices such as market segmentation and predictive modeling.

Comprehensively exploring the spatiotemporal aspects of PPL reservation data through geovisualizations can help managers learn about visitor usage. For example, exploring the geospatial demand distribution for groups of facilities within a destination region can help mangers better distribute use among facilities. Further, identifying communities that historically underutilized recreational opportunities at PPLs is the first step necessary in engaging those communities to increase participation. If the data is enriched by visitor origin geography, socioeconomic and geographic challenges that prevent citizens from accessing public lands can be identified. These insights into the spatiotemporal patterns of visitor demand from both the facility and visitor origins perspectives can help managers improve and promote visitor experiences for different segments of the population. These insights could help drive new planning and break down the inequities facing socially and economically underserved communities.

Unlike other countries, the number of visits to PPLs in the U.S. and Japan is decreasing (Balmford et al., 2009). Through geospatial segmentation and community profiling, this trend can be examined across different segments of the population. To support this and various other forms of geospatial analytics within the research community, we aim to publish a data set that includes the complete set of enrichments described in Sect. 2.3 for the U.S. Federal PPL data presented in this chapter. Hopefully providing access to a free cleaned data product will allow other research teams or PPL managers to explore different aspects of this historical record through the creation of geovisualizations, web-maps, models and forecasts. Ideally, this data will be used in support of making open spaces, parks, and public lands easier to reach and explore, so that more citizens can be part of the benefits that these lands afford society (myAmerica Developer Summit, 2015). Finally, web-maps that support visitation planning can help engage citizens and create our next generation of PPL visitors, supporters and advocates. 


\section{References}

ArcGIS for Desktop. (2016, February). Retrieved March 3, 2016, from http://desktop.arcgis.com/en/ ArcGIS Zip Code Layers. (2016, March 31). Retrieved March 31, 2016, from http://www.arcgis. com/home/search.html?q=zip\%20code\&restrict=true\&focus=layers

Bahaire, T., \& Elliott-White, M. (1999). The application of geographical information systems (GIS) in sustainable tourism planning: A review. Journal of Sustainable Tourism, 7(2), 159-174.

Balmford, A., Beresford, J., Green, J., Naidoo, R., Walpole, M., \& Manica, A. (2009). A global perspective on trends in nature-based tourism. PLoS Biol, 7(6), e1000144.

Beeco, J. A., Hallo, J. C., English, W. R., \& Giumetti, G. W. (2013). The importance of spatial nested data in understanding the relationship between visitor use and landscape impacts. Applied Geography, 45, 147-157. doi:10.1016/j.apgeog.2013.09.001.

Beeco, J. A., Hallo, J. C., \& Brownlee, M. T. J. (2014). GPS visitor tracking and recreation suitability mapping: Tools for understanding and managing visitor use. Landscape and Urban Planning, 127, 136-145. doi:10.1016/j.landurbplan.2014.04.002.

Bell, R. R., \& Zabriskie, N. B. (1978). Assisting marketing decisions by computer mapping: A branch banking application. Journal of Marketing Research, 15, 122-128.

Bishop, I. D., \& Gimblett, H. R. (2000). Management of recreational areas: GIS, autonomous agents, and virtual reality. Environment and Planning B: Planning and Design, 27(3), 423-435.

Center for Responsible Travel. (2016). The case for responsible travel: Trends \& statistics 2015. Washington, DC. https://ecotourism.app.box.com/s/rxiyp65744sqilmrybfk8mys3qvjbe9g

Chavez, D. J. (2001). Managing outdoor recreation in California: Visitor contact studies 19891998 (No. Gen. Tech. Rep. PSW-GTR-180.) (p. 100). Albany, NY: Pacific Southwest Research Station, Forest Service, U. S. Department of Agriculture.

Chen, R. J. (2007). Geographic information systems (GIS) applications in retail tourism and teaching curriculum. Journal of Retailing and Consumer Services, 14(4), 289-295.

Chhetri, P. (2015). A GIS methodology for modelling hiking experiences in the Grampians National Park, Australia. Tourism Geographies, 17(5), 795-814. doi:10.1080/14616688. 2015.1083609.

Chrisman, N. (2001). Reference systems for measurement. In Exploring geographical information systems (2nd ed., pp. 15-35). Wiley. http://www.citeulike.org/group/2170/article/1104881

Crnojevac, I., Gugić, J., \& Karlovčan, S. (2010). eTourism: A comparison of online and offline bookings and the importance of hotel attributes. Journal of Information and Organizational Sciences, 34(1), 41-54.

DeLappe, R. (2016, February 24). WebEx about data provided by the Recreation One-Stop Program [WebEx].

Elliott-White, M. P., \& Finn, M. (1997). Growing in sophistication: The application of geographical information systems in post-modern tourism marketing. Journal of Travel \& Tourism Marketing, 7(1), 65-84.

Every Kid in a Park. (2015, April 24). Retrieved March 16, 2016, from http://www.nationalparks. org/ook/every-kid-in-a-park

Gahegan, M. (2009). Visual exploration and explanation in Geography analysis with light. In Geographic data mining and knowledge discovery (2nd ed., pp. 291-324). Boca Raton, FL: CRC Press.

Gomez, L., Haesevoets, S., Kuijpers, B., \& Vaisman, A. A. (2009). Spatial aggregation: Data model and implementation. Information Systems, 34(6), 551-576. doi:10.1016/j.is.2009.03.002.

Gray, J., Chaudhuri, S., Bosworth, A., Layman, A., Reichart, D., Venkatrao, M., et al. (1997). Data cube: A relational aggregation operator generalizing group-by, cross-tab, and sub-totals. Data Mining and Knowledge Discovery, 1(1), 29-53. doi:10.1023/A:1009726021843. 
Haklay, M., Singleton, A., \& Parker, C. (2008). Web mapping 2.0: The neogeography of the GeoWeb. Geography Compass, 2(6), 2011-2039.

Hanink, D. M., \& Stutts, M. (2002). Spatial demand for national battlefield parks. Annals of Tourism Research, 29(3), 707-719. doi:10.1016/S0160-7383(01)00085-8.

Hanink, D. M., \& White, K. (1999). Distance effects in the demand for wildland recreational services: the case of national parks in the United States. Environment and Planning A, 31(3), 477-492. doi:10.1068/a310477.

Hirschey, J. (2014). Symbiotic relationships: Pragmatic acceptance of data scraping. Berkeley Technology Law Journal, 29. http://papers.ssrn.com/sol3/papers.cfm?abstract_id=2419167

Lansky, D. (2016, March 9). It's time for the Moneyball of destination marketing. Retrieved March 15, 2016, from https://www.tnooz.com/article/destination-marketing-analytics/

Loomis, J. (2004). How bison and elk populations impact park visitation: A comparison of results from a survey and a historic visitation regression model. Society \& Natural Resources, 17(10), 941-949. doi:10.1080/08941920490505338.

Manning, R. E. (2014). Research to guide management of outdoor recreation and tourism in parks and protected areas. Koedoe, 56(2), 1-7.

McAdam, D. (1999). The value and scope of geographical information systems in tourism management. Journal of Sustainable Tourism, 7(1), 77-92.

Mckercher, B., \& Lew, A. A. (2003). Distance decay and the impact of effective tourism exclusion zones on international travel flows. Journal of Travel Research, 42(2), 159-165.

Miller, F. L. (2008). Using a GIS in market analysis for a tourism-dependent retailer in the Pocono Mountains. Journal of Travel \& Tourism Marketing, 25(3-4), 325-340. doi:10.1080/ 10548400802508416.

Miller, H. J., \& Han, J. (2009). Geographic data mining and knowledge discovery: An overview. In Geographic data mining and knowledge discovery (pp. 1-26). Boca Raton, FL: CRC Press.

myAmerica Developer Summit. (2015, April). Retrieved April 6, 2016, from http://openglobe. github.io/myamerica-devsummit/

Neuvonen, M., Pouta, E., Puustinen, J., \& Sievanen, T. (2010). Visits to national parks: Effects of park characteristics and spatial demand. Journal for Nature Conservation, 18(3), 224-229. doi:10.1016/j.jnc.2009.10.003.

O'Connor, A., Zerger, A., \& Itami, B. (2005). Geo-temporal tracking and analysis of tourist movement. Mathematics and Computers in Simulation, 69(1-2), 135-150. doi:10.1016/j. matcom.2005.02.036.

Olston, C., \& Najork, M. (2010). Web crawling. Foundations and Trends in Information Retrieval, 4(3), 175-246.

Panel Discussion on Changing the Culture for Open Data, § Government Data Sharing Community of Practice. (2015). The Loft, 600 F Street NW, Washington, DC 2004. http://www.gao.gov/ assets/680/674021.pdf

Python. (2016). Retrieved April 4, 2016, from https://www.python.org/

Recreation Information Database-RIDB. (2016). Retrieved April 5, 2016, from https://ridb. recreation.gov/

Supak, S., Brothers, G., Bohnenstiehl, D., \& Devine, H. (2015). Geospatial analytics for federally managed tourism destinations and their demand markets. Journal of Destination Marketing \& Management, 4(3), 173-186.

Supak, S. K., Devine, H. A., Brothers, G. L., Rozier Rich, S., \& Shen, W. (2014). An open source web-mapping system for tourism planning and marketing. Journal of Travel \& Tourism Marketing, 31(7), 835-853.

Thelwall, M., \& Stuart, D. (2006). Web crawling ethics revisited: Cost, privacy, and denial of service. Journal of the American Society for Information Science and Technology, 57(13), $1771-1779$.

United Nations Environment Programme. (2011). Towards a green economy: Pathways to sustainable development and poverty eradication (p. 419). http://www.unep.org/greeneconomy/Por tals/88/documents/ger/ger_final_dec_2011/Green\%20EconomyReport_Final_Dec2011.pdf 
Van Berkel, D. B., Munroe, D. K., \& Gallemore, C. (2014). Spatial analysis of land suitability, hot-tub cabins and forest tourism in Appalachian Ohio. Applied Geography, 54, 139-148.

Wedel, M., \& Kamakura, W. A. (2000). Market segmentation: Conceptual and methodological foundations (Vol. 8). Boston: Springer. doi:10.1007/978-1-4615-4651-1.

Wolf, I. D., Wohlfart, T., Brown, G., \& Lasa, A. B. (2015). The use of public participation GIS (PPGIS) for park visitor management: A case study of mountain biking. Tourism Management, 51, 112-130. doi:10.1016/j.tourman.2015.05.003.

Zhao, J., Forer, P., Sun, Q., \& Simmons, D. (2013). Multiple-view strategies for enhanced understanding of dynamic tourist activity through geovisualization at regional and national scales. Cartography and Geographic Information Science, 40(4), 349-360. doi:10.1080/ 15230406.2013.783449. 